\title{
No spillover partial eigenvalue assignment in second-order linear systems using the Brauer's theorem and dense influ- ence matrices
}

\author{
Jessica Motta $^{1, *}$, Marconi de Almeida ${ }^{1, * *}$, Tito Santos ${ }^{1, * * *}$, and José Araújo ${ }^{2, * * * *}$ \\ ${ }^{1}$ Universidade Federal da Bahia, Departamento de Engenharia Elétrica, Rua Aristides Novis, 2, Feder- \\ ação, Salvador-BA, Brazil \\ ${ }^{2}$ Instituto Federal de Educação, Ciência e Tecnologia da Bahia, Rua Emídios dos Santos, S/N, Barbalho, \\ Salvador-BA, Brazil
}

\begin{abstract}
The control of vibrations for systems with second-order linear dynamics is a cornerstone for several engineering application, as structural vibration control in civil and aerospace structures. In such systems, sometimes only a few eigenvalues must be mitigated in situations as resonances, flutter, and another dangerous vibratory phenomenon. The use of active vibration control is an attractive alternative due to its flexibility and the availability of general design approaches. In the case of partial eigenvalue assignment, no spillover can be achieved, that is, only a small spectrum or eigenstructure partition is reallocated, with the remaining being unperturbed. In this paper, the no spillover property of Brauer's spectrum perturbation theorem is applied to compute a dense influence matrix structure of actuation, reassigning then the dangerous target eigenvalues and kept the remaining of the spectrum unchanged. A Sylvester equation must be solved using only the part of the spectrum to be reassigned. The results are tested in a benchmark taken from the specialized literature, and thus confirming its effectiveness.
\end{abstract}

\section{Introduction}

The control of vibrating structures is a remarkable field, with several applications in engineering, as civil structures - buildings, bridges, highways - aerospace industry, and another relevant examples [1],[2],[3],[4],[5],[6]. Such structures can be modeled in a versatile way by linear second-order differential equations, some of them that rise from finite element techniques - FEM [7] or from receptance approach [8],[9]. Often, the structures can experiment resonance, flutter and other dangerous vibrating phenomenon due to gust winds, air-streams or weight of human bodies. The use of active vibration control techniques (AVC) gives to the designer safety against that phenomenon, and the techniques to implement model-based AVC permits its application to globally suppress undesirable vibrations. Several works relate active vibration control techniques, and the pole/eigenstructure assignment is widely explored

\footnotetext{
*e-mail: Jessicalimamota@gmail.com

**e-mail: moliveiradealmeida1986@gmail.com

***e-mail: tlsantos@ufba.br

****e-mail: araujo@ieee.org
} 
[10],[11],[12]. In particular, the no spillover technique in pole/eigenvalue or eigenstructure assignment with discrete or dense influence matrix is an interesting approach, because only a small amount of information regarding the eigenstructure, that is, that must to be reassigned, is necessary for the available algorithms.

Recent works have applied the Brauer's Theorem, and it's versions [13],[14] to the pole placement in first-order systems [15]. Also, the model update and partial natural frequency assignment have been assessed in [16] using displacement feedback and in[17] using acceleration feedback. The Brauer's Theorem for perturbation over matrix is a milestone for the solution of certain no spillover designs. In this work, an algorithm for the design of no spillover eigenvalue assignment is proposed by using an alternative version of Brauer's Theorem. The left eigenvectors are explored, and a dense influence matrix is computed together with the velocity plus displacement feedback matrices. As is usual in the no spillover techniques, only the part of the spectrum to be reassigned must to be known, and the technique can be combined with other design requirements, as minimum norm or robustness. A numerical example is offered to illustrate the effectiveness of the approach.

\section{Preliminaries}

\subsection{Brauer's theorem}

Brauer's theorem and its generalizations or modifications have been developed in the last six decades since the original breakthrough. The Brauer's theorem for the perturbation of a single eigenvalue is stated below:

Theorem 2.1: Let $\mathbf{A}$ be a $n \times n$ arbitrary matrix with eigenvalues given by $\left\{\lambda_{1}, \lambda_{2}, \ldots, \lambda_{n}\right\}$. Let $\mathbf{x}_{k}$ be an eigenvector of $\mathbf{A}$ associated with the eigenvalue $\lambda_{k}$, and let $\mathbf{q} \in \mathbb{R}^{1 \times n}$ be an arbitrary row vector. Then the matrix $\mathbf{A}+\mathbf{x}_{1} \mathbf{q}$ has eigenvalues $\left\{\lambda_{1}, \lambda_{2}, \ldots, \lambda_{k}+\mathbf{q} \mathbf{x}_{1}, \ldots \lambda_{n}\right\}$.

This result reveals a simple manner to perturb a single eigenvalue with no spillover of the remaining spectrum. It is particularly useful in the development of the main results of this proposal.

\subsection{Second-order linear systems}

Second-order linear differential equations plays a central role in description of vibratory phenomena. Such class of systems serve to describe finite element models obtained form the discretization of distributed parameter systems or for lumped parameter vibrating systems. This particular approach is useful, for example, in structural dynamics and other mechanical or civil applications. The systems have the form:

$$
\mathbf{M} \ddot{\mathbf{x}}(t)+\mathbf{C} \dot{\mathbf{x}}(t)+\mathbf{K} \mathbf{x}(t)=\mathbf{B u}(t),
$$

where $\mathbf{M}, \mathbf{C}$ and $\mathbf{K}$ are, respectively, the mass, damping and stiffness matrices, each of them of order $n, \mathbf{x}(t)$ is the displacement vector, $\mathbf{B} \in \mathbb{R}^{n \times p}$ is an influence matrix that describes the action of the control input $\mathbf{u} \in \mathbb{R}^{p}$. The use of linear control for system (1) is conducted by the feedback of the displacement and velocity vector in the form:

$$
\mathbf{u}(t)=\mathbf{F}_{s} \dot{\mathbf{x}}(t)+\mathbf{G}_{s} \mathbf{x}(t)
$$

The system (1) can be expressed in an alternative, double-dimension state-space form as:

$$
\left[\begin{array}{c}
\dot{\mathbf{x}}(t) \\
\ddot{\mathbf{x}}(t)
\end{array}\right]=\left[\begin{array}{cc}
\mathbf{0} & \mathbf{I} \\
-\mathbf{M}^{-1} \mathbf{K} & -\mathbf{M}^{-1} \mathbf{C}
\end{array}\right]\left[\begin{array}{l}
\mathbf{x}(t) \\
\dot{\mathbf{x}}(t)
\end{array}\right]+\left[\begin{array}{c}
\mathbf{0} \\
\mathbf{M}^{-1} \mathbf{B}
\end{array}\right] \mathbf{u}(t)
$$


Regardless the disadvantage of this form due to the mass matrix inversion, it will be shown in the next section it advantages in the proposed solution.

\subsection{Statement of the problem}

In the present work, the main objective of applying the linear controller (2) in the system (1) is the well-known partial eigenvalue/eigenstructure assignment: given a set of resonant or unstable eigenvalues, says, $\lambda_{1}, \cdots, \lambda_{k}$, with $k \ll 2 n$, compute the feedback matrices $\mathbf{F}_{s}$ and $\mathbf{G}_{s}$ to reassign them to a new set $\mu_{1}, \cdots, \mu_{k}$, with the remaining of the spectrum $\lambda_{k+1}, \cdots, \lambda_{2 n}$ kept unperturbed. Several works in the last two decades have contributed to the solution of this problem with a fixed structure for the influence matrix $\mathbf{B}$ [18-20]. The proposal in this paper will be shown to hold some relevant features of these works, but taking into account the simplicity of the result derived from the Brauer's theorem by considering the influence matrix as part of the design, as in [10].

\section{Main results}

\subsection{A corollary based on Brauer's theorem}

Theorem 2.1 is now restated in an alternative form using the concept of left eigenvectors:

Corollary 3.1: Let $\mathbf{A}$ be a $n \times n$ arbitrary matrix with eigenvalues given by $\left\{\lambda_{1}, \lambda_{2}, \ldots, \lambda_{n}\right\}$. Let $\mathbf{y}_{k}$ be an left eigenvector of $\mathbf{A}$ associated with the eigenvalue $\lambda_{k}$, and let $\mathbf{q} \in \mathbb{R}^{n}$ be an arbitrary column vector. Then the matrix $\mathbf{A}+\mathbf{q} \mathbf{y}_{k}$ has eigenvalues $\left\{\lambda_{1}, \lambda_{2}, \ldots, \lambda_{k}+\mathbf{y}_{k} \mathbf{q}, \ldots \lambda_{n}\right\}$.

The proof is omitted here due to space limitations, but the reader car refer to some similar proofs as can be seen in [15],[12]. As in the case of Brauer's theorem, this result can be easily extended to the case in which one must to reassign multiple eigenvalues, in general complex eigenpairs. For that case, the eigenpairs to be ressigned, as well as the corresponding eigenvectors must be grouped in real representations following the notation:

- $\boldsymbol{\Lambda}_{1}=\operatorname{diag}\left(\left[\begin{array}{cc}\alpha_{1} & \beta_{1} \\ -\beta_{1} & \alpha_{1}\end{array}\right], \cdots,\left[\begin{array}{cc}\alpha_{l} & \beta_{l} \\ -\beta_{l} & \alpha_{l}\end{array}\right]\right)$, in which $\lambda_{k}=\operatorname{conj}\left(\lambda_{k+1}\right)=\alpha_{k}+i \beta_{k}, k=1, \ldots, 2 l$. It is a real representation of the eigenvalues that must be reassigned.

- $\boldsymbol{\Lambda}_{2}$ is the matrix containing the remaining of the spectrum of $\mathbf{A}$, such that $\sigma(\mathbf{A})=\sigma\left(\boldsymbol{\Lambda}_{1}\right) \cup$ $\sigma\left(\boldsymbol{\Lambda}_{2}\right)$.

- $\mathbf{Y}_{1}=\left[\begin{array}{lllll}\mathfrak{R} e\left(\mathbf{y}_{1}^{T}\right) & \mathfrak{J} m\left(\mathbf{y}_{1}^{T}\right) & \ldots & \mathfrak{R} e\left(\mathbf{y}_{l}^{T}\right) & \mathfrak{J} m\left(\mathbf{y}_{l}^{T}\right)\end{array}\right]^{T}$. It is a real representation of the left eigenvectors corresponding the the eigenvalues that must be reassigned.

By following this notation, and given an arbitrary matrix $\mathbf{Q} \in \mathbb{R}^{n \times 2 l}$, it can be shown that:

$$
\sigma\left(\mathbf{A}+\mathbf{Q} \mathbf{Y}_{1}\right)=\sigma\left(\mathbf{\Lambda}_{1}+\mathbf{Y}_{1} \mathbf{Q}\right) \cup \sigma\left(\mathbf{\Lambda}_{2}\right)
$$

\subsection{Application in partial eigenvalue assignment}

Now, Eq. (4), derived from the Corollary 3.1, is applied in the linear control design of the system (1) with feedback (2). The control law (2) can be considered with the description (3) in order to provide the following autonomous representation:

$$
\left[\begin{array}{c}
\dot{\mathbf{x}}(t) \\
\ddot{\mathbf{x}}(t)
\end{array}\right]=\left\{\left[\begin{array}{cc}
\mathbf{0} & \mathbf{I} \\
-\mathbf{M}^{-1} \mathbf{K} & -\mathbf{M}^{-1} \mathbf{C}
\end{array}\right]+\left[\begin{array}{c}
\mathbf{0} \\
\mathbf{M}^{-1} \mathbf{B}
\end{array}\right]\left[\begin{array}{ll}
\mathbf{G}_{s} & \mathbf{F}_{s}
\end{array}\right]\right\}\left[\begin{array}{l}
\mathbf{x}(t) \\
\dot{\mathbf{x}}(t)
\end{array}\right] .
$$


Then, consider that the set of eigenvalues and in a real form $\boldsymbol{\Lambda}_{1}$, with respective eigenvectors also in real form $\mathbf{Y}_{1}$. This set of eigenvalues can be reassigned in new position in the complex plane $\mathbb{C}$ if one sets:

$$
\tilde{\mathbf{A}}=\left[\begin{array}{cc}
\mathbf{0} & \mathbf{I} \\
-\mathbf{M}^{-1} \mathbf{K} & -\mathbf{M}^{-1} \mathbf{C}
\end{array}\right], \tilde{\mathbf{B}}=\left[\begin{array}{c}
\mathbf{0} \\
\mathbf{M}^{-1} \mathbf{B}
\end{array}\right] \equiv \mathbf{Q}, \mathbf{H}=\left[\begin{array}{ll}
\mathbf{G}_{s} & \mathbf{F}_{s}
\end{array}\right] \equiv \mathbf{Y}_{1} .
$$

Hence, the following procedure can be carried to compute the influence matrix $\mathbf{B}$ and the feedback gain matrices $\mathbf{F}_{s}$ and $\mathbf{G}_{s}$ :

\section{Procedure 3.1}

1. Given the system matrices $\mathbf{K}, \mathbf{C}, \mathbf{M}$, compute the matrix $\tilde{\mathbf{A}}$, and get the undesired eigenvalues in a real form $\boldsymbol{\Lambda}_{1} \in \mathbb{R}^{p \times p}$, with respective real form left eigenvectors $\mathbf{Y}_{1} \in \mathbb{R}^{p \times 2 n}$.

2. Compute $\mathbf{G}_{s}$ and $\mathbf{F}_{s}$, by taking a partition of the left eigenvectors as:

$$
\mathbf{Y}_{1}=\left[\begin{array}{ll}
\mathbf{Y}_{11} & \mathbf{Y}_{12}
\end{array}\right]
$$

with the first $n$ and the last $n$ first columns of $\mathbf{Y}_{1}$ :

$$
\mathbf{G}_{s}=\mathbf{Y}_{11}, \mathbf{F}_{s}=\mathbf{Y}_{12}
$$

3. Set the target eigenvalues into a real form $\overline{\boldsymbol{\Lambda}}_{1}$; set an arbitrary rank- $p$ matrix $\boldsymbol{\Gamma} \in \mathbb{R}^{n \times p}$; compute the solution $\mathbf{T} \in \mathbb{R}^{p \times p}$ for the Lyapunov-Sylvester equation:

$$
\boldsymbol{\Lambda}_{1} \mathbf{T}-\mathbf{T} \overline{\mathbf{\Lambda}}_{1}=-\mathbf{Y}_{12} \mathbf{\Gamma} .
$$

4. Compute the influence matrix:

$$
\mathbf{B}=\mathbf{M} \boldsymbol{\Gamma} \mathbf{T}^{-1}
$$

To give a brief outline on the Steps 3 and 4 of the procedure, recall that if the matrices $\Lambda_{1}+\mathbf{Y}_{1} \mathbf{Q}=\boldsymbol{\Lambda}_{1}+\mathbf{Y}_{12} \mathbf{M}^{-1} \mathbf{B}$ and $\bar{\Lambda}_{1}$ have the same eigenvalues, then, there exists a nonsingular matrix $\mathbf{T}$ such that:

$$
\mathbf{T}^{-1}\left(\boldsymbol{\Lambda}_{1}+\mathbf{Y}_{12} \mathbf{M}^{-1} \mathbf{B}\right) \mathbf{T}=\bar{\Lambda}_{1} \Leftrightarrow \boldsymbol{\Lambda}_{1} \mathbf{T}-\mathbf{T} \overline{\mathbf{\Lambda}}_{1}=-\mathbf{Y}_{12} \mathbf{\Gamma}
$$

with $\boldsymbol{\Gamma}=\mathbf{M}^{-1} \mathbf{B} \mathbf{T}$.

Remark 3.1: In the procedure, the free parameter $\boldsymbol{\Gamma}$ offers flexibility in the final value of the influence matrix. Then, the designer can explore the choice to gives, as an instance, the minimum norm for $\mathbf{B}$ or robustness of the closed-loop eigenvalues [21].

Remark 3.2: Despite some possible drawbacks, for instance, the necessity of explicitly inverting the mass matrix and the use of one column in the influence matrix for each eigenvalue to be reassigned, the main advantages of the method are that only the eigenstructure to be assigned must be known, as in work [10]. Moreover, no constraints are imposed on the system matrices, as symmetry or positiveness.

\section{Numerical example}

Consider the model of a wing in airstream, for which the flutter phenomenon is observed in open-loop [22],[23]:

$$
\mathbf{M}=\left[\begin{array}{ccc}
17.600 & 1.280 & 2.890 \\
1.280 & 0.824 & 0.413 \\
2.890 & 0.413 & 0.725
\end{array}\right], \mathbf{C}=\left[\begin{array}{lll}
7.660 & 2.450 & 2.100 \\
0.230 & 1.040 & 0.223 \\
0.600 & 0.756 & 0.658
\end{array}\right], \mathbf{K}=\left[\begin{array}{ccc}
121.000 & 18.900 & 15.900 \\
0 & 2.700 & 0.145 \\
11.900 & 3.640 & 15.500
\end{array}\right]
$$


The open-loop eigenvalues of the system are $\lambda_{1,2}=-0.8848 \pm j 8.4415, \lambda_{3,4}=0.0947 \pm$ $j 2.5229$ and $\lambda_{5,6}=-0.9180 \pm j 1.7606$. A critical eigenpair $\lambda_{3,4}$ is present, that explains the flutter phenomenon with dangerous, increasing vibrations in the structure. The proposed approach is applied in order to reassign this eigenpair to the new positions $\mu_{3,4}=$ $-0.2000 \pm j 2.5229$. For the sake of comparison, the method is applied first with an arbitrary choice of $\Gamma$, and later, the minimization of the Frobenius norm for the influence matrix is conducted with the initial value to $\Gamma$ being the previous value. Thus, by taking $\Gamma_{0}^{T}=\left[\begin{array}{ccc}1.5301 & -0.2490 & -1.0642 \\ 1.6035 & 1.2347 & -0.2296\end{array}\right]$, the following results for the control matrices are obtained:

$$
\mathbf{B}=\left[\begin{array}{ll}
9.9651 & 29.3294 \\
0.1478 & 2.5942 \\
1.3247 & 4.8364
\end{array}\right], \mathbf{F}=\left[\begin{array}{lll}
0.0701 & 0.0517 & 0.0247 \\
0.3500 & 0.0327 & 0.0613
\end{array}\right], \mathbf{G}=\left[\begin{array}{ccc}
0.8986 & 0.1522 & 0.1701 \\
0 & -0.0692 & -0.0112
\end{array}\right]
$$

At last, the minimization problem: $\min _{\Gamma}\|\mathbf{B}(\Gamma)\|_{F}$ is carried using Eqs. (9) and (10), and the influence matrix is then computed results:

$$
\mathbf{B}=\left[\begin{array}{cc}
-0.0150 & 2.3544 \\
8.3597 & 0.6221 \\
0.1797 & 1.2378
\end{array}\right]
$$

The results of the eigenvalue assignment are displayed in Table 1. Both the designs are effective to provide the control goals. The design with the minimum norm is better in the sense of economic design, spent less energy of the control effort than that for the static design of $\mathbf{B}$.

Table 1. No spillover design of wing in airstream flutter control

\begin{tabular}{ccc}
\hline & $\Gamma=\Gamma_{0}$ & $\Gamma=\arg \min _{\Gamma}\|\mathbf{B}(\Gamma)\|_{F}$ \\
\cline { 2 - 3 } & $-0.8848+8.4415 i$ & $-0.8848+8.4415 i$ \\
$-0.8848-8.4415 i$ & $-0.8848-8.4415 i$ \\
Assigned & $-0.2000+2.5229 i$ & $-0.2000+2.5229 i$ \\
Eigenvalues & $-0.2000-2.5229 i$ & $-0.2000-2.5229 i$ \\
$-0.9180+1.7606 i$ & $-0.9180+1.7606 i$ \\
$-0.9180-1.7606 i$ & $-0.9180-1.7606 i$ \\
\hline $\begin{array}{c}\text { Influence Matrix } \\
\text { Norm }\end{array}$ & $\|\mathbf{B}\|_{F}=31.4867$ & $\|\mathbf{B}\|_{F}=8.7965$ \\
\hline $\begin{array}{c}\text { Precision of the Reassigned } \\
\text { Eigenvalues }\end{array}$ & $4.8850 e-15$ & $3.8240 e-15$ \\
\hline $\begin{array}{c}\text { Maximum Spillover of the } \\
\text { Unperturbed Eigenvalues }\end{array}$ & $3.7750 e-15$ & $1.9860 e-15$ \\
\hline
\end{tabular}

\section{Concluding remarks}

A new approach to partial eigenvalue assignment is proposed inspired by the Brauer's results and its modifications. The new method is capable of reassigning a set of undesired eigenvalues by computing the feedback matrices for velocity and displacement and the influence matrix of actuators in a vibrating system. The method works in systems with 
matrices without any constraints, as symmetry or definiteness. A procedure to implement the method is given, and an example helps to illustrate its effectiveness in a flutter control design. Future works include the development of the method using acceleration and velocity feedback, a somewhat attractive alternative to the feedback presented in this paper.

The authors would like to thanks to IFBA/PRPGI for the grants - \#09/2018.

\section{References}

[1] L. Meirovitch, Y. Sharony, Journal of Optimization Theory and Applications 69, 31 (1991)

[2] T. Nakamura, Y. Nakamura, Journal of Structural Engineering (United States) 119, 50 (1993)

[3] S.H. Strogatz, D.M. Abrams, A. McRobie, B. Eckhardt, E. Ott, Nature 438, 43 (2005)

[4] R. Plaut, Journal of Sound and Vibration 309, 613 (2008)

[5] Y. Li, Y.Y. Qiu, F.J. Peng, Active Vibration Control for the Flexible Spacecraft Structure Based on an ATMD/PPF Strategy, in Vibration, Structural Engineering and Measurement I (Trans Tech Publications, 2012), Vol. 105 of Applied Mechanics and Materials, pp. 668-674

[6] C.J. Kim, Journal of Mechanical Science and Technology 31, 4145 (2017)

[7] R.R. Craig, A.J. Kurdila, Fundamentals of Structural Dynamics, 2nd Edition (Wiley \& Sons, 2006), ISBN 0471430447

[8] J.E. Mottershead, Y.M. Ram, AIAA Journal 45, 562 (2007)

[9] J.E. Mottershead, M.G. Tehrani, S. James, Y.M. Ram, Journal of Sound and Vibration 311, 1391 (2008)

[10] B. Datta, S. Elhay, Y. Ram, D. Sarkissian, Journal of Sound and Vibration 230, 101 (2000)

[11] M.G. Tehrani, J. Mottershead, IFAC Proceedings Volumes 45, 1174 (2012)

[12] J.M. Araújo, Mechanical Systems and Signal Processing 98, 368 (2018)

[13] A. Brauer, Duke Math. J. 19, 75 (1952)

[14] R.L. Soto, O. Rojo, Linear Algebra and its Applications 416, 844 (2006)

[15] R. Bru, R. Cantó, R.L. Soto, A.M. Urbano, Central European Journal of Mathematics 10, 312 (2012)

[16] J. Zhang, J. Ye, H. Ouyang, X. Yin, Journal of Low Frequency Noise, Vibration \& Active Control (2018), disponible in https://doi.org/10.1177/1461348418756026

[17] J. M.Araújo, T.L. Santos, Proceedings of the Institute of Mechanical Engineers, Part I: Joornal of Systems and Control Engineering (2018), doi 10.1177/0959651818771482

[18] B.N. Datta, S. Elhay, Y.M. Ram, Linear Algebra and its Applications 257, 29 (1997)

[19] Z. Bai, B.N. Datta, J. Wang, Mechanical Systems and Signal Processing 24, 766 (2010)

[20] J.M. Araújo, C.E. Dórea, L.M. Gonçalves, J.B. Carvalho, B.N. Datta, Journal of Low Frequency Noise, Vibration and Active Control 37, 253 (2018)

[21] S. Brahma, B. Datta, Journal of Sound and Vibration 324, 471 (2009)

[22] D. Henrion, M. Sebek, V. Kucera, Kybermetika 41, 1 (2005)

[23] T.H. Abdelaziz, \{ISA $\}$ Transactions 57, 71 (2015) 